\title{
A Uniqueness result for a generalized Radon transform
}

\author{
B. L. FRIDMAN \\ Mathematics Department \\ Wichita State University \\ Wichita, KS 67260-0033 \\ FRIDMAN@TWSUVM.UC.TWSU.EDU
}

March1994

\begin{abstract}
There exist five families of Lipschitz curves on the unit square such that any continuous function is uniquely defined by the values of its integral (properly defined) along these curves. We present this uniqueness result as a consequence of the Kolmogorov's superposition theorem.
\end{abstract}

\section{Introduction ${ }^{1,2}$}

The main intention of this article is to demonstrate a connection of results in Linear Superpositions of functions and the uniqueness problem for a generalized Radon transform. We will actually present here one such relation, namely, a consequence of the Kolmogorov's superposition theorem. We will start with a description of a general problem.

Suppose that for each $\alpha \in J$ ( $J$ is the set of indices) there is a family (also called a spread) $\Omega_{\alpha}$ of non-intersecting submanifolds $\Gamma_{t}^{\alpha}, t \in T=T_{\alpha}$, of

\footnotetext{
${ }^{1}$ Key words. Radon transform, spreads, uniqueness, superposition

${ }^{2} \mathrm{AMS}(\mathrm{MOS})$ subject classifications. 65R10, 44A12, 92C55, 26B40
} 
some manifold $M=\bigcup_{t \in T} \Gamma_{t}^{\alpha}$ and for each $\Gamma=\Gamma_{t}^{\alpha}$ there is a measure $d \mu_{\Gamma}$ so one can introduce a generalized Radon transform by

$$
R f(\Gamma)=\int_{\Gamma} f d \mu_{\Gamma}
$$

for an integrable function $f$. Typically $\Gamma_{t}^{\alpha}$ are smooth hypersurfaces, the parameter set $T$ is a one-dimensional interval, $M \subseteq \mathbf{R}^{\mathbf{n}}$, and $f$ is a continuous function with compact support. The general uniqueness (or invertibility of $R$ ) problem we are considering here is this. If $R f\left(\Gamma_{t}^{\alpha}\right)=R f(\alpha, t)=0$ for all $\alpha, t$ then $f=0$. Similar problems have been addressed in a number of papers (see [1]-[6], [9]-[10], [12]-[16]).

The question we will discuss here is the following one: how many families (the cardinality of $J$ ) does it take to assure the uniqueness (invertibility) of $R$ ? Intuitively it seems to be very likely that if the number of different families is infinite the uniqueness takes place and if the number of families is finite the uniqueness does not hold. In many cases that have been considered this assertion is supported. One however should recall the result of J. Boman [1] showing that the uniqueness is not necessarily assured in case $\Gamma_{t}^{\alpha}$ are straight lines in $\mathbf{R}^{2}, \alpha \in J=[0,2 \pi]$ is infinite, and the measure is $C^{\infty}$ and positive.

Here we will be considering the case of a finite number of families. Is it possible to find such a finite system of families that uniqueness holds for continuous functions? Unexpectedly the answer to this question in a typical case is positive. The main result of this paper stated below is the following one. There exist five families of Lipschitz curves on the unit square in $\mathbf{R}^{\mathbf{2}}$ and such a measure on each of these curves that the uniqueness property holds for all continuous functions.

For the special case we are describing here we will have $\Gamma=\Gamma_{t}^{\alpha}$ will be curves on the closure of the unit square $\Delta \subset \mathbf{R}^{2}$, we also will use the notation $I_{\Gamma}(f)$ instead of $R f(\Gamma)$ for the corresponding integral (1). One more remark. In many cases it is desirable to consider such natural measures $d \mu_{\Gamma}$ that the Fubini's theorem holds: "double" integral of $f$ over $M$ could be presented as a repeated integral, that is an integral of $I_{\Gamma_{t}^{\alpha}}(f)$ over $T^{\alpha}$ with some measure $d \mu_{\alpha}(t)$. The measure we will be introducing below is such a natural measure (see (3)).

As stated above this result is going to be a consequence of the well-known 
theorem by A.N. Kolmogorov, theorem that presented a solution of the 13-th problem of Hilbert. We use the notation $\Delta$ for the closure of the unit square: $\Delta=\{(x, y) \mid 0 \leq x, y \leq 1\}$.

Theorem A. (Superposition Theorem of A. N. Kolmogorov). There exist five functions $\Phi_{i}(x, y)=\varphi_{i}(x)+\psi_{i}(y), i=1, \ldots 5$, such that for any $f \in C(\Delta)$ there exist continuous functions $\chi_{i}(t)$ so that

$$
f(x, y)=\sum_{i=1}^{5} \chi_{i}\left(\varphi_{i}(x)+\psi_{i}(y)\right) .
$$

The functions $\varphi_{i}(x), \psi_{i}(y)$ can be chosen to be strictly increasing and Lip1 functions.

For proof see [11], the choice of Lipschitz functions was proved in [7].

\section{Definitions and basic results}

A reasonable way to introduce a family of curves is to consider them as level sets of a function. Following a suggestion of L. Ehrenpreis such a family will be called a spread and the corresponding function will be a spread function. We will present the definition in two steps.

1)Let $D$ be a domain in $\mathbf{R}^{2}$ and $\Phi \in C(\bar{D})$. Consider $\Gamma_{t}=\{(x, y) \mid \Phi(x, y)=$ $t\} \cap \bar{D}$ - the level curve of $\Phi$, and $[a, b]=\Phi(\bar{D})$ the range of $\Phi$. Clearly $\Gamma_{t} \cap \Gamma_{\tau}=\emptyset$ if $t \neq \tau$ and $\bar{D}=\bigcup_{t \in[a, b]} \Gamma_{t}$. The set $\Omega=\left\{\Gamma_{t} \mid t \in[a, b]\right\}$ we will call a spread on $\bar{D}$ and $\Phi$ the spread function.

2)A spread $\Omega$ on $\bar{D}$ generated by a spread function $\Phi$ is called a proper spread if the following holds. There exists a homeomorphism $\phi: \bar{D} \rightarrow \bar{U}=$ $\phi(\bar{D})$ such that the set $\left\{\phi\left(\Gamma_{c}\right) \mid \Gamma_{c} \in \Omega\right\}$ is a set of straight parallel lines on $\bar{U}: \phi\left(\Gamma_{t}\right) \| \phi\left(\Gamma_{\tau}\right)$ for any $t, \tau \in[a, b]$.

All results that follow hold for many domains in $R^{2}$. Without any significant loss of generality and for simplicity of exposition we will consider our domain to be the unit square $\Delta$. 
Suppose we have a proper spread $\Omega$ of curves on $\Delta$ generated by a continuous function $\Phi$ and $\Gamma=\Gamma_{t} \in \Omega$. Let $f \in C(\Gamma)$. Then $f$ can be extended to a continuous function on $\Delta$ which we will still denote by $f$. We now will introduce $I_{\Gamma}(f)$ the integral of $f$ over $\Gamma$. Let $\Gamma(\epsilon)=\{(x, y) \in \Delta \mid \Phi(x, y) \in$ $(t-\epsilon, t+\epsilon)\}$. Then we define

$$
I_{\Gamma}(f)=\lim _{\epsilon \rightarrow 0} \frac{\int_{\Gamma(\epsilon)} f d A}{m e s_{2}(\Gamma(\epsilon))},
$$

where $d A$ is the area element and $m e s_{2}$ is the Lebesgue area.

Example. One can check the following. If $\Phi \in C^{1}(\Delta)$ and $\operatorname{grad} \Phi \neq 0$ on $\Gamma=\Gamma_{t}$ with positive length, then $I_{\Gamma}(f)=\frac{\int_{\Gamma} f /\|\operatorname{grad} \Phi\| d s}{l_{g}(\Gamma)}$, where $d s$ is the element of length and $l_{g}(\Gamma)$ is the weighted length of $\Gamma ; l_{g}(\Gamma)=\int_{\Gamma} \frac{1}{\|\operatorname{rad} \Phi\|} d s$.

Lemma 1. 1.Let $\Gamma$ from (2) be fixed. If $I_{\Gamma}(f)$ exists for some continuous extension of $f$ from $\Gamma$ to $\Delta$, then it exists and has the same value for any such continuation of $f$ to $\Delta$. In this case $\left|I_{\Gamma}(f)\right| \leq \max _{x \in \Gamma}|f(x)|$.

2. The set $E(\Gamma)$ of continuous functions on $\Gamma$ for which $I_{\Gamma}(f)$ exists, forms a closed linear subspace in $C(\Gamma) . I_{\Gamma}(f)$ is a linear bounded functional on $E(\Gamma)$.

3. If $f=c=$ const. on $\Gamma$ then $I_{\Gamma}(f)=c$.

4. If $\chi(t) \in C[a, b]$ then $I_{\Gamma t}(\chi(\Phi(x, y))=\chi(t)$.

Proof. First one can check 3. It will follow from the fact that the extension is continuous and from the mean value theorem for the Lebesgue integral on bounded functions.

One can now check 1 . The first assertion follows from the following observation. The difference between two extensions of $f$ is zero on $\Gamma$ and therefore the integral of the difference will always exist and be equal to zero. One can prove the inequality by using the standard inequalities for the integral of a bounded function.

2. follows from the linear property of Lebesgue integral and the already proved inequality in 1 . 
4. follows from 3 .

Denote by $\Phi_{*}$ the push-forward induced by the function $\Phi: \Delta \rightarrow \Phi(\Delta)=$ $[a, b]=J$. (The push-forward takes measures on $\Delta$ to measures on $J$ ). We now introduce a countably additive measure $\mu$ on $J$ by the formula $\mu(d t)=$ $\Phi_{*}(d A)$ (that is: $\mu(T)=\operatorname{mes}_{2}\left(\Phi^{-1}(T)\right)$ for $\left.T \subseteq J\right)$. One can see that $\mu$ is a countably additive measure on $J$, and all Borel sets on $J$ are $\mu$ measurable. Let $f \in C(\Delta)$. We may now consider a measure $\nu_{f}$ given by $\nu_{f}(d t)=\Phi_{*}(f d A)$. Clearly $\nu_{f}$ must be absolutely continuous with respect to $\mu$. Therefore by the Radon-Nikodim theorem there exists $g \in L_{1}(\mu)$ such that $\nu_{f}=g \mu$, where

$$
g(t)=\lim _{\epsilon \rightarrow 0} \frac{\left.\nu_{f}(t-\epsilon, t+\epsilon)\right)}{\mu(t-\epsilon, t+\epsilon)}=\lim _{\epsilon \rightarrow 0} \frac{\int_{\Gamma_{t}(\epsilon)} f d A}{\operatorname{mes}_{2}\left(\Gamma_{t}(\epsilon)\right)}
$$

and $g(t)$ exists almost everywhere with respect to $\mu$-measure (a.e. $\mu$ ) on $J$. If one compares this formula with the definition $(2)$ of $I_{\Gamma}(f)$, one has the proof of the first part of the following:

Lemma 2. 1. $g(t)=I_{\Gamma_{t}}(f)$, a.e. $\mu$, and therefore the last integral is uniquely defined for a fixed $f$ a.e. $\mu$ on $J$. This is to say that the union of all level curves $\Gamma$ for which the limit in (2) does not exist, forms a set of Lebesgue area zero.

2. The following formula holds:

$$
\int_{\Delta} f d A=\int_{a}^{b} I_{\Gamma t}(f) \mu(d t)
$$

Proof of the last formula follows from the following observation. Considering measures as linear functionals on the space of continuous functions we know that the push-forward $\Phi_{*}$ is the adjoint of the pull-back $\Phi^{*}: u \longmapsto u \circ \Phi$ for $u \in C(J)$, that is,

$$
\langle v, u \circ \Phi\rangle=\left\langle v, \Phi^{*}(u)\right\rangle=\left\langle\Phi_{*}(v), u\right\rangle .
$$

Taking $u=1$ and $v=f d A$ we obtain (3). 
Below we will say that a certain property holds for almost every $\Gamma$ if the union $S$ of all $\Gamma$ for which this property fails has Lebesgue area zero: $\mathrm{mes}_{2}(S)=0$. Obviously this definition does not depend on the spread function generating the set of curves; it depends on the set of curves (the spread) only.

The space $E(\Gamma)$ (see Lemma 1 ) is nonempty and $I_{\Gamma}(f)$ is a bounded linear functional on this space. Obviously $E(\Gamma) \subseteq C(\Gamma)$.

Lemma 3. $E(\Gamma)=C(\Gamma)$ for almost every $\Gamma$ ( a.e. $\Gamma$ in the above defined meaning).

Proof. Let set $S$ be the union of all such $\Gamma$ for which $E(\Gamma) \neq C(\Gamma)$. Consider all polynomials of two variables with rational coefficients. They form a countable set $\left\{P_{1}, P_{2}, ..\right\}$. Let $S_{n}$ be the union of all such $\Gamma$ for which $I_{\Gamma}\left(P_{n}\right)$ does not exist. By the previous Lemma 2. $m e s_{2}\left(S_{n}\right)=0$. Since every continuous function can be approximated uniformly on $\Delta$ by this set of polynomials, $S \subseteq \bigcup_{n=1}^{\infty} S_{n}$, and therefore $\operatorname{mes}_{2}(S)=0$.

Lemma 4. If $f \in C(\Delta)$ and $\chi(t) \in C[a, b]$ then for almost all (relative to the $\mu$ measure) $t \in[a, b]$

$$
I_{\Gamma_{t}}\left\{f(x, y) \chi(\Phi(x, y)\}=I_{\Gamma_{t}}(f) \chi(t) .\right.
$$

Proof follows from the linear property of the introduced integral and the uniqueness of it from Lemma 2.

\section{The main statement}

Now we are ready to prove the following

Theorem B. There exist five proper spreads $\Omega_{i}, i=1, \ldots, 5$ of curves on $\Delta$ such that if $f \in C(\Delta)$ and $I_{\Gamma}(f)=0$ for almost every curve $\Gamma$ in any $\Omega_{i}$ 
then $f \equiv 0$.

Remark. Almost every $\Gamma$ means as before: all $\Gamma$ with the exception of a set of curves whose union has zero area in $\Delta$.

Proof. Consider $\Phi_{i}(x, y)=\phi_{i}(x)+\psi_{i}(y)$ from Theorem A. As noted above all these functions can be chosen to be strictly increasing and satisfy the Lipschitz condition. We can also assume that $\phi_{i}(\Delta)=\left[0, q_{i}\right], \psi_{i}(\Delta)=\left[0, p_{i}\right]$, so $\Phi(\Delta)=\left[0, q_{i}+p_{i}\right]$. Consider now $\Gamma_{t}^{i}=\left\{(x, y) \in \Delta \mid \Phi_{i}(x, y)=t\right\}$.

1. First we prove that $\Omega_{i}=\left\{\Gamma_{t}^{i}\right\}$ is a proper spread. Consider the system

$$
\begin{aligned}
& t=\phi_{i}(x)+\psi_{i}(y) \\
& u=\phi_{i}(x)-\psi_{i}(y)
\end{aligned}
$$

One can show by using the monotonicity of the functions involved that the above system provides a homeomorphism of $\Delta$ onto the rectangle $0 \leq$ $t+u \leq 2 q_{i}, 0 \leq t-u \leq 2 p_{i}$ and therefore $\Omega_{i}$ is in one-to-one correspondence with parallel lines $t=$ const.

2. Let $f \in C(\Delta)$ be a function for which $I_{\Gamma_{t}^{i}}(f)=0$ for almost all $\Gamma_{t}^{i}$. According to Theorem A, $f(x, y)=\sum_{i=1}^{5} \chi_{i}\left(\Phi_{i}(x, y)\right)$. Consider now (we use (3) and (4))

$$
\begin{gathered}
\iint_{\Delta} f^{2}(x, y) d A=\iint_{\Delta} f(x, y) \sum_{i=1}^{5} \chi_{i}\left(\Phi_{i}(x, y)\right) d A= \\
\sum_{i=1}^{5} \int_{0}^{q_{i}+p_{i}} I_{\Gamma_{t}^{i}}\left\{f(x, y) \chi_{i}\left(\Phi_{i}(x, y)\right\} \mu_{i}(d t)=\right. \\
=\sum_{i=1}^{5} \int_{0}^{q_{i}+p_{i}} I_{\Gamma_{t}^{i}}(f) \chi_{i}(t) \mu_{i}(d t)=0
\end{gathered}
$$

Therefore $f \equiv 0$.

Remark 1. The constructed spreads $\Omega_{i}$ consist of Lipschitz rectifiable curves. The $I_{\Gamma}(f)$ is not necessarily absolutely continuous for every $\Gamma$. 
Problem. Will the statement of Theorem B hold if $I_{\Gamma}(f)=\int_{\Gamma} f d s$, where $d s$ is the element of the arc length?

Remark 2. Theorem A (see [11]) was proved for a general case in $R^{n}$ for all $n$. Similarly one can prove a statement analogous to Theorem B in case $n>2$, replacing curves by surfaces correspondingly.

Remark 3. One can check that the construction in (2) and most that followed can be done for $f \in L_{1}(\Delta)$ instead of a continuous function. This would lead to

Theorem $\mathbf{B}^{\prime}$. There exist five proper spreads $\Omega_{i}, i=1, \ldots, 5$ of curves on $\Delta$ such that if $f \in L_{1}(\Delta)$ and $I_{\Gamma}(f)=0$ for almost every curve $\Gamma$ in any $\Omega_{i}, i=1, \ldots, 5$, then $f=0$.

To prove this, one can repeat the proof presented above and consider $\iint f g d A, g \in C(\Delta)$. Then one can proceed as above to prove that this integral is zero and thus $f$ is orthogonal to any continuous function. Therefore $f$ is equal to zero in $L_{1}$.

The following statement shows the critical difference in the outcome if we replace the Lipschitz condition for functions $\Phi_{i}$ generating our families of curves, by the requirement that these functions be continuously differentiable.

Theorem C. Let spreads $\Omega_{i}, i=1, \ldots, s$ of curves on $\Delta$ be generated by spread functions $\Phi_{i} \in C^{1}(\Delta)$. Then there exists a function $f \in L_{2}(\Delta), f \neq 0$, such that $I_{\Gamma}(f)=0$ for almost every curve $\Gamma$ in any $\Omega_{i}, i=1, \ldots, s$.

Proof follows from the result in [8], Thm.4, that proves that the set $G=\left\{g \mid g(x, y)=\sum_{i=1}^{s} \chi_{i}\left(\Phi_{i}(x, y)\right), \chi_{i} \in C\right\}$ is nowhere dense in $L_{2}(\Delta)$. Taking now $f \in L_{2}(\Delta), f \perp G$, one can check by using the same idea as above that $I_{\Gamma}(f)=0$ for almost every $\Gamma$ in any $\Omega_{i}$.

Acknowledgment. I am very grateful to Professor Peter Kuchment for valuable discussions and encouragement. I am also most thankful to the 
referee for useful suggestions that improved the exposition. 


\section{References}

[1] J. Boman, An example of non-uniqueness for a generalized Radon transform, Journal d'Analyse Mathematique, 61 (1993), pp. 395-399.

[2] J. Boman, Uniqueness theorems for generalized Radon transforms, in Constructive Theory of Functions '84, Sofia, 1984, pp. 173-176.

[3] J. Boman, Helgason's support theorem for Radon transforms - a new proof and a generalization, Mathematical methods in Tomography, Proceedings of a conference held at Oberwolfach 1990, Springer Lecture Notes in Mathematics (no. 1497).

[4] J. Boman and E. T. Quinto, Support theorems for real-analytic Radon transforms, Duke Math. J. 55 (1987), pp. 943-948.

[5] J. Boman and E. T. Quinto, Support theorems for Radon transform on real-analytic line complexes in three-space, Trans. Amer. Math. Soc. 335 (2) (1993), pp. 877-890.

[6] D. V. Finch, Uniqueness of the attenuated X-ray transform in the physical range, Inverse Problems 2 (1986), pp. 197-203.

[7] B. L. Fridman, Improvement in the smoothness of functions in the Kolmogorov superposition theorem, Dokl. Akad. Nauk SSSR 177 (1967), pp. 1019-1022= Soviet Math. Dokl. 8 (1967), pp. 1550-1553.

[8] B. L. Fridman, An estimate of the dimension of the null spaces of linear superpositions, Mat.Sb. 82 (124) (1970), pp. 111-125 = Math. USSR Sb. 11 (1970), pp. 101-114.

[9] A. Greenleaf and G. Uhlmann, Non-local inversion formulas for the Xray transform, Duke Math. J. 58 (1989), pp. 205-240.

[10] S. Helgason, Groups and geometric analysis, Academic Press, New York, 1984. 
[11] A. N. Kolmogorov, On the representation of continuous functions of many variables by superposition of continuous functions of one variable and addition, Dokl. Akad. Nauk SSSR 114 (1957), pp. 953-956 = Amer. Math. Soc. Transl. (2) 28 (1963), pp. 55-59.

[12] A. Markoe and E. T. Quinto, An elementary proof of local invertibility for generalized and attenuated Radon transforms, SIAM J. Math. Anal. 16 (1985), pp. 1114-1119.

[13] R. G. Mukhometov, The problem of recovery of a two-dimensional Riemannian metric and integral geometry, Dokl. Akad. Nauk SSSR, 232 no. 1 (1977), pp. 32-35 = Soviet Math. Dokl. 18 no. 1 (1977), pp. 27-31.

[14] R.G. Mukhometov, A problem of reconstructing a Riemannian metric, Sibirsk. Mat. Zh. 22 no. 3 (1981), pp. 119-135 = Siberian Math. J. 22 no. 3 (1981), pp. 420-433.

[15] E. T. Quinto, The invertibility of rotation invariant Radon transforms, J. Math. Anal. Appl. 91 (1983), pp. 510-522.

[16] L. Zalcman, Uniqueness and nonuniqueness for the Radon transform, Bull. London Math. Soc. 14 (1982), pp. 241-245. 